\title{
Robust Speaker Clustering using Mixtures of von Mises-Fisher Distributions for Naturalistic Audio Streams
}

\author{
Harishchandra Dubey, Abhijeet Sangwan, John H. L. Hansen \\ Robust Speech Technologies Lab, Center for Robust Speech Systems \\ The University of Texas at Dallas, Richardson, TX- 75080, USA \\ \{Harishchandra.Dubey, Abhijeet.Sangwan, John.Hansen\}@utdallas.edu
}

\begin{abstract}
Speaker Diarization (i.e. determining who spoke and when?) for multi-speaker naturalistic interactions such as Peer-Led Team Learning (PLTL) sessions is a challenging task. In this study, we propose robust speaker clustering based on mixture of multivariate von Mises-Fisher distributions. Our diarization pipeline has two stages: (i) ground-truth segmentation; (ii) proposed speaker clustering. The ground-truth speech activity information is used for extracting i-Vectors from each speechsegment. We post-process the i-Vectors with principal component analysis for dimension reduction followed by lengthnormalization. Normalized i-Vectors are high-dimensional unit vectors possessing discriminative directional characteristics. We model the normalized i-Vectors with a mixture model consisting of multivariate von Mises-Fisher distributions. K-means clustering with cosine distance is chosen as baseline approach. The evaluation data is derived from: (i) CRSS-PLTL corpus; and (ii) three-meetings subset of AMI corpus. The CRSSPLTL data contain audio recordings of PLTL sessions which is student-led STEM education paradigm. Proposed approach is consistently better than baseline leading to upto $44.48 \%$ and $53.68 \%$ relative improvements for PLTL and AMI corpus, respectively.
\end{abstract}

Index Terms: Speaker clustering, von Mises-Fisher distribution, Peer-led team learning, i-Vector, Naturalistic Audio.

\section{Introduction}

Speaker Diarization attempts to answer who spoke and when? in an audio stream [1]. Domains involving practical application of speaker diarization are understanding and transcription of broadcast news, audio-recorded meetings, telephonic conversations etc [2] 3]. The challenges in speaker diarization is application-dependent. NIST Rich Transcription evaluations focused on broadcast news and meetings audio while NIST SRE evaluations had summed-channel telephone data [4]. Speaker diarization pipeline consists of several components such as speech activity detection (SAD) [5, 6], speaker change detection, speaker clustering, and re-segmentation [4]. Among these, speaker clustering is the main component.

This paper propose a model-based speaker clustering using $\mathrm{i}$-Vectors. The target application is naturalistic audio streams from Peer-Led Team Learning (PLTL) sessions [7] 8]. PLTL is

This material is presented to ensure timely dissemination of scholarly and technical work. Copyright and all rights therein are retained by the authors or by the respective copyright holders. The original citation of this paper is: H. Dubey, A. Sangwan, J. H. L. Hansen, "Robust Speaker Clustering using Mixtures of von Mises-Fisher Distributions for Naturalistic Audio Streams", ISCA INTERSPEECH, Sept. 2-6, 2018, Hyderabad, India. a student-led STEM education model where a peer-leader facilitate problem-solving among 6-8 students [9] 10]. Previously, we developed speech systems for automatic extraction of behavioral metrics related to PLTL sessions such as dominance, word-count and student participation etc [10, 11].

\section{Background}

Given the importance of robust clustering in speaker diarization, several approaches were developed such as agglomerative hierarchical clustering (AHC) [12], top-down clustering [13], Spherical K-means clustering [14] etc. Authors proposed joint speaker segmentation and clustering scheme [4]. In [15], the MAP-adapted Gaussian mixture-models (GMMs) were combined with Bayesian information criterion (BIC) for speaker diarization. A reduced complexity clustering approach leverages modified integer linear programming (ILP) [16]. Unsupervised calibration of PLDA scores was used for i-Vector clustering on CALLHOME corpus [17]. Previously, von Mises-Fisher distribution were used for text-independent speaker identification based on line spectral frequencies (LSFs) features [18]. The von Mises-Fisher distributions were employed in several applications such as similarity measure for text-snippets [19], bioinformatics [20] etc.

\section{Front-end}

In this study, our diarization pipeline consists of three stages: (i) speech dereverberation; (ii) ground-truth speech segmentation; and (iii) proposed speaker clustering based on mixture of von Mises-Fisher distributions (movMF). As the focus on this paper is to develop robust speaker clustering, we used ground-truth speech segmentation information. Using ground-truth speech activity detection is important to prevent irrelevant errors due to incorrect segmentation. Previously, researchers found that speaker clustering could be developed independent of other components in diarization pipeline [21].

\subsection{Speech Enhancement and Ground-truth Segmentation}

The CRSS-PLTL data is significantly reverberated (see section 5.2.2 so we perform experiments with both original (raw) and de-reverb audio. We employed weighted prediction error (WPE)-based dereverberation approach [22]. After dereverberation, we get the speaker segments using the groundtruth speech activity labels. From each segment, i-Vector was extracted followed by PCA dimension reduction and lengthnormalization. We used only raw audio from AMI meeting corpus to avoid reporting too many results. 


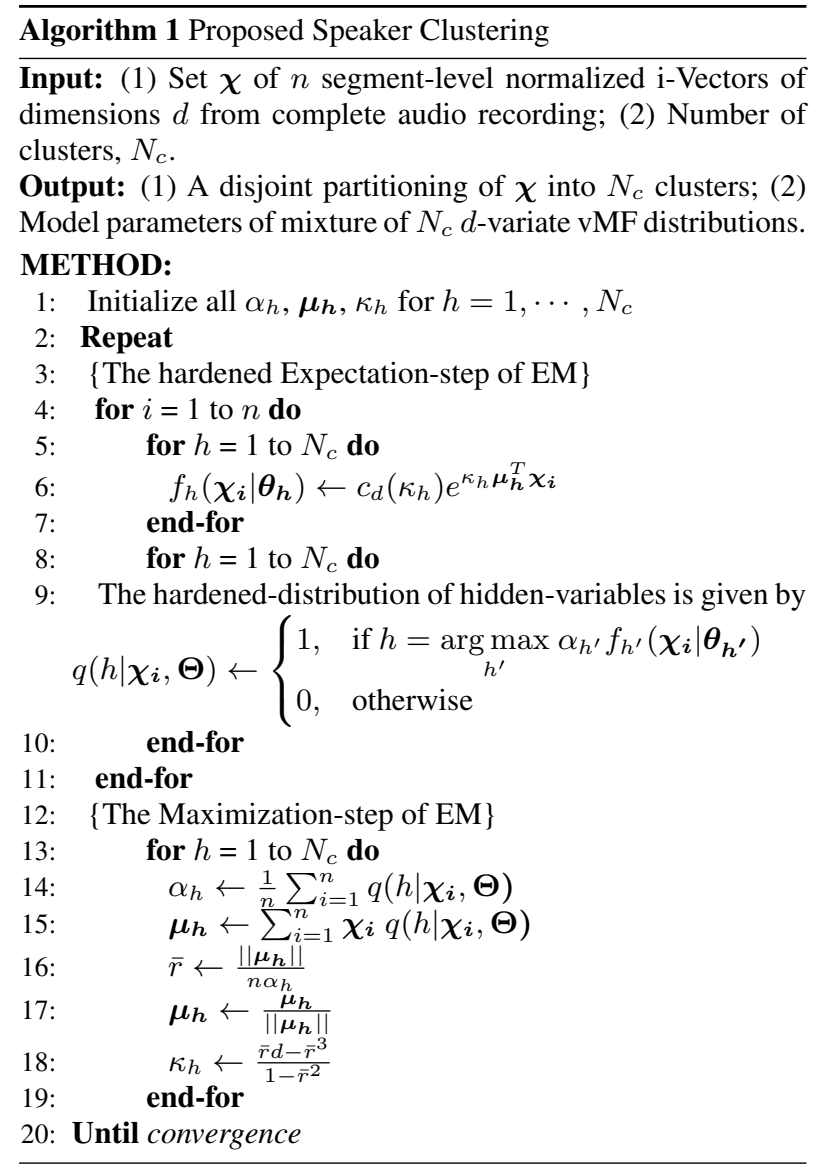

\section{2. i-Vector Speaker Model}

Diarization involve extracting i-Vectors from short speech segments (typically one second) unlike speaker verification where complete utterance is used. Numerous techniques were developed for clustering i-Vectors using cosine similarity [23, 24]. The i-Vector framework combined the speaker and channel variability sub-spaces of linear distortion model into a totalvariability space represented by matrix $\mathbf{T}[25,26]$. A speakerand-session-dependent GMM super-vector, $\mathbf{S}$ is decomposed as

$$
\mathbf{S}=\mathbf{S}_{\mathbf{u b m}}+\mathbf{T w}
$$

where $\mathbf{S}_{\mathbf{u b m}}$ is the Universal Background Model (UBM) supervector [25]. The latent variables, $\mathbf{w}$ are i-Vectors. The totalvariability matrix $\mathbf{T}$ is a low-rank projection matrix that maps high-dimensional speaker super-vectors to low-dimensional total-variability space [25, 26]. We use frame-level 20-MFCC features extracted from $40 \mathrm{~ms}$ windows at $10 \mathrm{~ms}$ skip-rate. A UBM with 512 components was trained for i-Vector extraction [25]. Given the short speaker-segments in PLTL, we choose the i-Vector dimension as 75 . We post-processed the segmentlevel i-Vectors with PCA for dimension reduction followed by length-normalization [27]. For rest of this paper, normalized i-Vectors refer to length-normalized ones.

\section{Proposed Speaker Clustering}

Speaker clustering for PLTL sessions is a challenging task due to following reasons: (i) overlapped-speech; (ii) skewed clusters in feature space; (iii) significant reverberation and multiple noise sources etc. We input the number of clusters in proposed approach. We model normalized i-Vectors from an audio stream with a mixture of $N_{c}$ multivariate ( $d$-variate) von Mises-Fisher distributions (movMF). Here, $N_{c}$ is number of speakers and $d$ is the i-Vector dimension. Previously, researchers analyzed the advantages of $i-V e c t o r$ normalization for speaker modelling [27]. Normalized i-vectors are high dimensional data lying on unit hypersphere. There are no closed form solutions for movMF parameters. However, it is possible to get reliable estimates of movMF parameters if input data is high-dimensional [28]. Normalized i-Vectors have significantly higher dimensions (e.g. 75) as compared to number of speaker (e.g. 8), thus movMF model could be approximated reliably for i-Vector clustering. The vMF distribution defines a probability density function (PDF) of data lying on unit hypersphere. For modeling the normalized i-Vectors with movMF model, we have a weight parameter, $\alpha$ for each vMF distribution. We adopt expectationmaximization (EM) algorithm for approximating the maximum likelihood (ML) estimates of movMF model developed in [28].

\subsection{EM-based Estimation of Model Parameters}

In this section, we summarize the approach for approximating the modal parameters using EM algorithm. We estimate the movMF model iteratively using normalized i-Vectors as detailed in Algorithm 1. The PDF of a $d$-variate vMF distribution is given by

$$
f(\boldsymbol{x} \mid \boldsymbol{\mu}, \kappa)=c_{d}(\kappa) e^{\kappa \boldsymbol{\mu}^{T} \boldsymbol{x}}
$$

where $\|\boldsymbol{\mu}\|=1, \kappa \geq 0$ and $d \geq 2$. Here, input data lies on unit hypersphere $\boldsymbol{x} \in \mathbb{S}^{d-1}$ and $(\cdot)^{T}$ denote transpose operation. Normalizing constant, $c_{d}(\kappa)$ is expressed as

$$
c_{d}(\kappa)=\frac{\kappa^{\frac{d}{2}-1}}{(2 \pi)^{\frac{d}{2}} I_{\frac{d}{2}-1}(\kappa)}
$$

where $I_{r}(\cdot)$ is modified Bessel function of first-kind and order $r$. PDF $f(\boldsymbol{x} \mid \boldsymbol{\mu}, \kappa)$ has two parameters, mean direction-vector $\boldsymbol{\mu}$, and concentration parameter $\kappa$. The $\kappa$ indicate how strongly the normalized i-Vectors drawn according to $f(\boldsymbol{x} \mid \boldsymbol{\mu}, \kappa)$ distribution lie near the mean direction-vector on unit hypersphere. Large $\kappa$ shows substantial concentration near $\boldsymbol{\mu}$. Consider a mixture of $N_{c} d$-variate vMF distributions as a generative model for normalized i-Vectors from an audio recording. Let $f_{h}\left(\boldsymbol{x} \mid \boldsymbol{\theta}_{\boldsymbol{h}}\right)$ denote the $h$-th vMF distribution in mixture model and its parameter-vector is $\boldsymbol{\theta}_{\boldsymbol{h}}=\left(\boldsymbol{\mu}_{\boldsymbol{h}}, \kappa_{h}\right)$ for $1 \leq h \leq N_{c}$. Then, the PDF of this mixture model is given as

$$
f(\boldsymbol{x} \mid \boldsymbol{\Theta})=\sum_{h=1}^{N_{c}} \alpha_{h} f_{h}\left(\boldsymbol{x} \mid \boldsymbol{\theta}_{\boldsymbol{h}}\right)
$$

where $\boldsymbol{\Theta}=\left\{\alpha_{1}, \cdots, \alpha_{N_{c}}, \boldsymbol{\theta}_{\mathbf{1}}, \cdots, \boldsymbol{\theta}_{N_{c}}\right\}$; and $\alpha_{h}$ are nonnegative weights such that $\sum_{h=1}^{N_{c}} \alpha_{h}=1$. Let $\chi=$ $\left\{\chi_{1}, \cdots, \chi_{n}\right\}$ be the stream of normalized i-Vectors modeled with mixture-model in Eq. 4 . Let $\boldsymbol{\zeta}=\left\{\zeta_{1}, \cdots, \zeta_{n}\right\}$ be the corresponding set of hidden variables that indicate the componentvMF distribution from which an i-Vector is sampled. Particularly, $\zeta_{i}=h$ if $\chi_{i}$ is sampled from distribution $f_{h}\left(\boldsymbol{x} \mid \boldsymbol{\theta}_{\boldsymbol{h}}\right)$. In terms of hidden-variable vector $\boldsymbol{\zeta}$, the $\log$-likelihood (LL) of $n$ observed i-Vectors is given by

$$
\ln \{P(\boldsymbol{\chi}, \boldsymbol{\zeta} \mid \boldsymbol{\Theta})\}=\sum_{i=1}^{n} \ln \left\{\alpha_{\zeta_{i}} f_{\zeta_{i}}\left(\boldsymbol{\chi}_{i} \mid \boldsymbol{\theta}_{\zeta_{i}}\right)\right\} .
$$

For a given $(\chi, \Theta)$, it is possible to estimate the most likely conditional distribution of $\zeta \mid(\chi, \Theta)$, and this forms the 


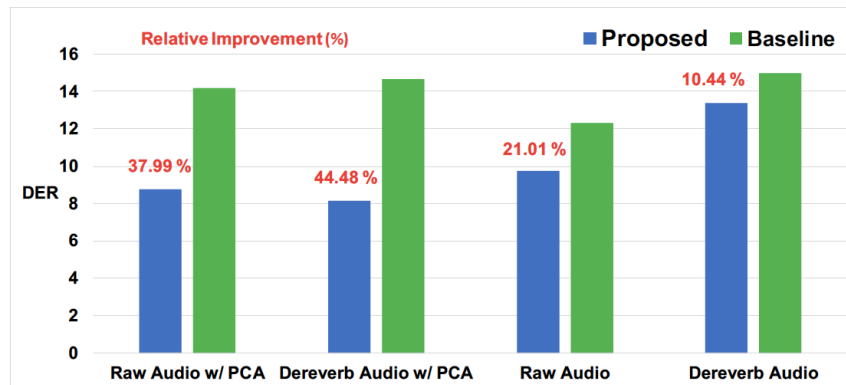

(a)

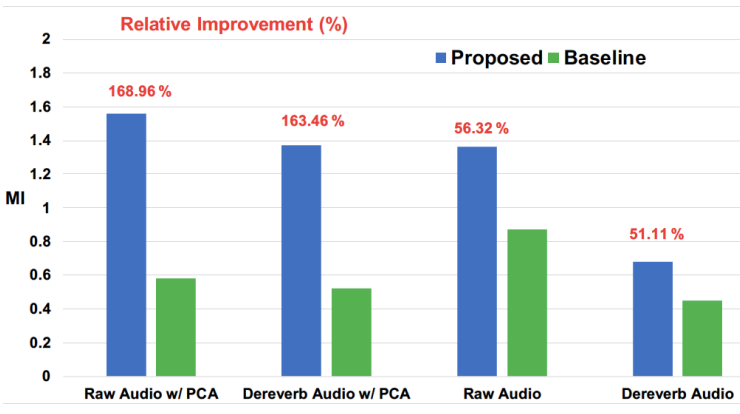

(b)

Figure 1: PLTL results: (a) Diarization error rate (DER) for proposed and baseline approaches. We used raw (original) and dereverbed audio in our experiments. The " $w / P C A "$ denotes PCA-based dimension reduction of $i$-Vectors to 51 dimensions before lengthnormalization. Relative reduction (\%) in DER with respect to baseline is shown in red color on top of each bar. (b) Frame-wise mutual information (MI) for proposed and baseline approaches. Relative increase (\%) in MI with respect to baseline is shown in red color above each bar.

Expectation-step in EM algorithm. Next, we use the EM algorithm for maximizing the expectation of Eq. 5 with constraints $\boldsymbol{\mu}_{h}^{T} \boldsymbol{\mu}_{\boldsymbol{h}}=1$ and $\kappa_{h} \geq 0$. As a result, we get the following expressions for movMF model parameters [28]:

$$
\begin{gathered}
\alpha_{h}=\frac{1}{n} \sum_{i=1}^{n} p\left(h \mid \chi_{\boldsymbol{i}}, \boldsymbol{\Theta}\right), \\
\boldsymbol{r}_{\boldsymbol{h}}=\sum_{i=1}^{n} \boldsymbol{\chi}_{\boldsymbol{i}} p\left(h \mid \boldsymbol{\chi}_{\boldsymbol{i}}, \boldsymbol{\Theta}\right), \\
\hat{\boldsymbol{\mu}}_{h}=\frac{\boldsymbol{r}_{\boldsymbol{h}}}{\left\|\boldsymbol{r}_{\boldsymbol{h}}\right\|}, \\
\frac{I_{\frac{d}{2}}\left(\hat{\kappa}_{h}\right)}{I_{\frac{d}{2}-1}\left(\hat{\kappa}_{h}\right)}=\frac{\left\|\boldsymbol{r}_{\boldsymbol{h}}\right\|}{\sum_{i=1}^{n} p\left(h \mid \boldsymbol{\chi}_{\boldsymbol{i}}, \boldsymbol{\Theta}\right)} .
\end{gathered}
$$

The Eqs. 8 and 9 correspond to Maximization-step in EM algorithm providing expressions for modal parameters. Next, given these parameters we consider updating the distribution of $\zeta \mid(\chi, \Theta)$ (i.e., an Expectation-step) to maximize the likelihood of i-Vectors given the estimated parameters from Eqs. 8 and 9

Since computing $\hat{\kappa}$ involves ratio of Bessel functions (see Eq. 9, it is not possible to obtain an analytic solution. Various numerical and/or asymptotic methods are used for approximating $\hat{\kappa}$. In this paper, we used the $\hat{\kappa}$ estimates developed in [28]. It is given as

$$
\hat{\kappa}=\frac{\bar{r} d-\bar{r}^{3}}{1-\bar{r}^{2}} .
$$

\subsection{Proposed Speaker Clustering: Algorithm 1}

We outline the proposed approach in Algorithm 1 It outputs the maximum-likelihood (ML) estimates of movMF parameters. This method essentially iterate over two steps in standard EM algorithm until it converges. In each Expectation-step, iVectors are hard-assigned to a cluster. The distribution of hidden variables is given by Step 9 of Algorithm 1 The i-Vectors are hard-assigned to a unique cluster on the basis of derived posterior distribution. Cluster assignment is done by computing the arg max over posteriors for each i-Vector (Step 9).

Next in Maximization-step of EM, the model parameters of component-vMFs are updated using the component-vMF distributions given the i-Vectors (Step 12 to Step 19 in Algorithm 1).

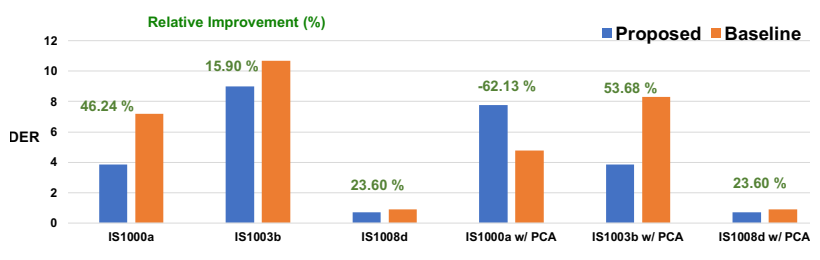

Figure 2: AMI (three-meetings subset) results: DER for proposed and baseline approaches. The " $w / P C A "$ case refers to PCA-based dimension reduction of $i$-Vectors to 65 before length-normalization. Relative reduction (\%) in DER with respect to baseline is shown in green color above each bar.

In the proposed hard-assignment approach, the posterior probabilities are restricted to have only binary i.e., 0 or 1 values. With hard-assignments, the distribution of hidden-variables is restricted to assume probability value 1 for some mixture component and 0 for all others. This strategy maximizes a lowerbound on incomplete log-likelihood (LL) of i-Vectors [28]. In other words, expectation over distribution $q(\cdot)$ lower bounds the LL of i-Vectors.

Upon convergence, the Algorithm 1 outputs the movMF model parameters and $N_{c}$ clusters of i-Vector data (Step 20). The hard-assignments in Step 9 reduce the computational complexity as posterior probabilities are binary values. Proposed clustering approach in Algorithm 1 requires only $\mathcal{O}\left(N_{c}\right)$ computations in each EM iteration. It need to store only the cluster assignments for all i-Vectors (i.e., $n$ integers). These two facts make the proposed speaker clustering computationally efficient and scalable.

\section{Experiments, Results \& Discussions}

\subsection{Baseline System}

The cosine similarity was previously used for comparing iVectors in K-means and mean-shift clustering [24, 23]. In this paper, we adopt spherical K-means (with cosine similarity) as baseline approach [29]. Spherical K-means projects the estimated cluster centroids onto unit hypersphere at the end of each maximization-step unlike conventional K-means. Spherical Kmeans is a special case of proposed movMF-based speaker clustering. If we impose all mixture weights ( $\alpha_{h}$ for $1 \leq h \leq N_{c}$ ) 
to be equal and all concentration parameters $\left(\kappa_{h}\right.$ for $1 \leq h \leq$ $N_{c}$ ) to be equal (with any value), then the proposed movMFbased speaker clustering becomes equivalent to spherical Kmeans.

\subsection{Evaluation Data}

\subsubsection{AMI Corpus}

Augmented Multi-party Interaction (AMI) corpus has multimodal data from meeting scenarios with reference speaker annotations. We derived our AMI evaluation set from audio recordings of multi-speaker interactions in three meetings. We used headset audio data for our experiments. We chose a three-meetings subset containing sessions IS1000a (26 min.), IS1003b (27 min.) and IS1008d (24 min.) from the popular 12-meetings subset of AMI corpus [30,31]. Each of the three meetings has four speakers discussing the design of a new remote control device.

\subsubsection{CRSS-PLTL Corpus}

Each PLTL session has a peer-leader to facilitate discussions among 6-8 students. PLTL model is known to enhance student's learning [32]. Typically, peer-leaders have previously passed the course with good grades. It is implemented for undergraduate STEM courses in U.S. universities. In association with UT-Dallas Student Success Center, we collected the CRSS-PLTL corpus [11, 8, 10, 6, 9] from five teams attending a chemistry course over eleven weeks. The weekly sessions were organized for approximately 70-80 minutes. Each participant wore a LENA device (with not-so-close microphone) for collecting naturalistic audio [9, 33]. In this manner, we collected multi-stream audio for each session (number of streams was same as total participants). The salient features of this data are: (i) many segments with overlapped-speech; (ii) short conversational-turns; (iii) multiple noise-sources; and (iv) significant reverberation. These factors made PLTL speaker diarization challenging. In this paper, we choose the channel corresponding to PLTL leader for diarization evaluation.

\subsection{Evaluation Metric}

We used two evaluation metrics namely: (i) diarization error rate (DER); and (ii) frame-level mutual information (MI) for scoring the system-output with respect to reference annotation. DER was introduced in the NIST Rich Transcription Spring 2003 evaluation (RT-03S). It is defined as the total percentage of reference speaker-time that is not correctly attributed to a speaker. Mathematically, DER [34] is given as:

$$
\mathrm{DER}=\frac{\phi_{f a}+\phi_{\text {miss }}+\phi_{\text {err }}}{\phi_{\text {total }}}
$$

where $\phi_{\text {total }}$ is the total time of all reference-segments, $\phi_{f a}$ is the system speaker-time not attributed to the reference speaker, $\phi_{m i s s}$ is the total reference speaker-time not attributed to a system speaker, and $\phi_{e r r}$ is the total reference speaker-time attributed to a wrong speaker. Unlike NIST RT evaluations [35], we do not apply any forgiveness collar to the reference human annotations prior to scoring. We consider overlapped-speech as an additional cluster while scoring the system outputs and during human annotations. We adopted the NIST md-eval scoring script (version-22) for DER computations [34].

MI quantifies the statistical similarity between frame-level system output and ground-truth. We incorporate overlappedspeech in MI computation. First of all, both ground-truth and system output are converted to $10 \mathrm{~ms}$ frame-level labels. Then, the frame-level MI (in bits) between system output and groundtruth is mathematically defined as:

$$
\mathrm{MI}=\sum_{i=1}^{R} \sum_{j=1}^{S} \frac{n_{i j}}{N} \log _{2} \frac{n_{i j} N}{r_{i} s_{j}},
$$

where $R, S$ are the number of reference and system clusters, respectively; $n_{i j}$ is the number of frames assigned to $i$-th reference and $j$-th system cluster; $r_{i}, s_{j}$ are the number of frames assigned to $i$-th reference, and $j$-th system cluster, respectively; and $N$ is the total number of frames. We compute MI values using the scoring scripts from the First DIHARD Challenge Evaluation [36].

\subsection{Results}

In this study, the PLTL data contains audio of the peer-leader's channel from a 80-minute session with seven students. We obtain the ground-truth segmentation and speaker labels from human annotators. Figure 1 compares the performance of proposed approach with baseline on PLTL data. Sub-figure 1 (a) illustrates the DER while (b) shows the frame-level MI for raw and dereverbed audio. The majority of PLTL speakerturns were less than one-second (though few lasted over twoseconds), we chose 75 (lower) dimensional i-Vectors. We repeated all experiments with PCA for reducing the i-Vector dimension to 51. This PLTL audio contains significantly $10 \%$ overlapped-speech that was incorporated as a separate cluster during evaluation. Thus, the number of cluster, $N_{c}=9$ that includes peer-leader, seven students and overlapped-speech. The proposed approach has improved performance in terms of lower DER and higher MI values compared to baseline. The consistent improvement in all cases with original and enhanced audio, with or without PCA validate the efficacy of movMF model for robust speaker clustering. We have similar observation on three-meetings subset of AMI data as illustrated in Fig. 2 We included only DER for AMI data to avoid presenting too many results. The CRSS-PLTL audio has higher levels and more varied forms of distortions as compared to AMI corpus resulting in a challenging diarization scenario. The proposed clustering approach has the ability to adapt the concentration parameter $\kappa$ for each component-vMF distribution in the mixture-model. This created a flexible modeling of normalized i-Vectors that is substantially better than spherical K-means as spherical K-means do not estimate the weight or concentration parameters unlike movMF model. The movMF clustering do a better job by taking advantage of the concentration estimates for each componentvMF distribution.

\section{Conclusions}

This paper proposed modeling of length-normalized i-Vectors with a mixture of multi-variate von Mises-Fisher distributions (movMF). Standard EM algorithm was used for estimating the model parameters. The normalized i-Vectors are highdimensional data lying on unit hypersphere that facilitated reasonable approximation of movMF model parameters. In general, movMF parameters do not have closed form solutions. The model parameters are leveraged for robust speaker clustering. The evaluation data was derived from naturalistic CRSS-PLTL and AMI meeting corpus. Accurate modeling resulted in improved performance of movMF speaker clustering compared to baseline spherical K-means. 


\section{References}

[1] S. E. Tranter and D. A. Reynolds, "An overview of automatic speaker diarization systems," IEEE Trans. on Audio, Speech, and Language Processing, vol. 14, no. 5, pp. 1557-1565, 2006.

[2] M. Huijbregts and D. A. van Leeuwen, "Large-scale speaker diarization for long recordings and small collections," IEEE Trans. on Audio, Speech, and Language Processing, vol. 20, no. 2, pp. 404-413, 2012.

[3] J. H. L. Hansen, H. Dubey, A. Sangwan, L. Kaushik, and V. Kothapally, "UTDallas-PLTL: Advancing multi-stream speech processing for interaction assessment in peer-led team learning,' The Journal of the Acoustical Society of America, vol. 143, no. 3, pp. 1869-1869, 2018.

[4] X. Anguera, S. Bozonnet, N. Evans, C. Fredouille, G. Friedland, and O. Vinyals, "Speaker diarization: A review of recent research," IEEE Trans. on Audio, Speech, and Language Processing, vol. 20, no. 2, pp. 356-370, 2012.

[5] H. Dubey, A. Sangwan, and J. H. L. Hansen, "Robust feature clustering for unsupervised speech activity detection," in IEEE ICASSP, 2018, pp. 2726-2730.

[6] — "Leveraging Frequency-Dependent Kernel and DIP-based Clustering for Robust Speech Activity Detection in Naturalistic Audio Streams," IEEE/ACM Trans. on Audio, Speech and Language Processing, pp. -, 2018.

[7] J. H. L. Hansen, K. Hickman, N. Jones, H. Dubey, A. Sangwan, and V. Kothapally, "UTDallas-PLTL: Leveraging Spoken Language Technology for Assessment of Communication based Learning Behavior in Peer-Led Team Learning," Sixth Annual Conference Peer-Led Team Learning International Society, Northeastern Illinois University, Chicago, Illinois, USA., pp. 5$10,2017$.

[8] H. Dubey, L. Kaushik, A. Sangwan, and J. H. L. Hansen, "A speaker diarization system for studying peer-led team learning groups," in ISCA INTERSPEECH, 2016, pp. 2180-2184.

[9] J. H. L. Hansen, J. Alberte, N. Jones, H. Dubey, and A. Sangwan, "Multi-stream audio analysis for knowledge extraction and understanding of small-group interactions in peer-led team learning," Seventh Annual Conference Peer-Led Team Learning International Society, the University of Texas at Dallas, Richardson, TX, USA, pp. 1-1, 2018.

[10] H. Dubey, A. Sangwan, and J. H. L. Hansen, "A robust diarization system for measuring dominance in peer-led team learning groups," in IEEE Spoken Language Technology Workshop (SLT), 2016, pp. 319-323.

[11] _ _ "Using speech technology for quantifying behavioral characteristics in peer-led team learning sessions," Computer Speech \& Language, vol. 46, pp. 343-366, 2017.

[12] H. Sun, B. Ma, S. Z. K. Khine, and H. Li, "Speaker diarization system for RT07 and RT09 meeting room audio," in IEEE ICASSP, 2010, pp. 4982-4985.

[13] S. Meignier, D. Moraru, C. Fredouille, J.-F. Bonastre, and L. Besacier, "Step-by-step and integrated approaches in broadcast new speaker diarization," Computer Speech \& Language, vol. 20, no. 2-3, pp. 303-330, 2006.

[14] J. H. Hansen, A. Sangwan, A. Ziaei, H. Dubey, L. Kaushik, and C. Yu, "Prof-Life-Log: Monitoring and assessment of human speech and acoustics using daily naturalistic audio streams," The Journal of the Acoustical Society of America, vol. 140, no. 4, pp. 3010-3010, 2016.

[15] X. Zhu, C. Barras, S. Meignier, and J. Gauvain, "Combining speaker identification and BIC for speaker diarization," in ISCA INTERSPEECH, 2005, pp. 2441-2444.

[16] G. Dupuy, S. Meignier, P. Deléglise, and Y. Estéve, "Recent Improvements on ILP-based Clustering for Broadcast News Speaker Diarization," in ISCA Odyssey, 2014, pp. 187-193.

[17] G. Sell and D. Garcia-Romero, "Speaker diarization with PLDA i-vector scoring and unsupervised calibration," in IEEE Spoken Language Technology Workshop (SLT), 2014, pp. 413-417.
[18] J. Taghia, Z. Ma, and A. Leijon, "On von-Mises Fisher mixture model in text-independent speaker identification," in ISCA INTERSPEECH, 2013, pp. 2499-2503.

[19] M. Sahami and T. D. Heilman, "A web-based kernel function for measuring the similarity of short text snippets," in Proceedings of the 15th international conference on World Wide Web. ACM, 2006, pp. 377-386.

[20] K. V. Mardia, C. C. Taylor, and G. K. Subramaniam, "Protein bioinformatics and mixtures of bivariate von Mises distributions for angular data," Biometrics, vol. 63, no. 2, pp. 505-512, 2007.

[21] M. Sinclair and S. King, "Where are the challenges in speaker diarization?" in IEEE ICASSP, 2013, pp. 7741-7745.

[22] T. Yoshioka, T. Nakatani, M. Miyoshi, and H. G. Okuno, "Blind separation and dereverberation of speech mixtures by joint optimization," IEEE Trans. on Audio, Speech, and Language Processing, vol. 19, no. 1, pp. 69-84, 2011.

[23] M. Senoussaoui, P. Kenny, T. Stafylakis, and P. Dumouchel, "A study of the cosine distance-based mean shift for telephone speech diarization," IEEE Trans. on Audio, Speech and Language Processing, vol. 22, no. 1, pp. 217-227, 2014.

[24] F. Castaldo, D. Colibro, E. Dalmasso, P. Laface, and C. Vair, "Stream-based speaker segmentation using speaker factors and eigenvoices," in IEEE ICASSP, 2008, pp. 4133-4136.

[25] N. Dehak, P. J. Kenny, R. Dehak, P. Dumouchel, and P. Ouellet, "Front-end factor analysis for speaker verification," IEEE Trans. on Audio, Speech, and Language Processing, vol. 19, no. 4, pp. 788-798, 2011.

[26] J. H. L. Hansen and T. Hasan, "Speaker recognition by machines and humans: A tutorial review," IEEE Signal Processing Magazine, vol. 32, no. 6, pp. 74-99, 2015.

[27] D. Garcia-Romero and C. Y. Espy-Wilson, "Analysis of i-vector length normalization in speaker recognition systems," in ISCA INTERSPEECH, 2011, pp. 249-252.

[28] A. Banerjee, I. Dhillon, J. Ghosh, and S. Sra, "Clustering on the unit hypersphere using von Mises-Fisher distributions," Journal of Machine Learning Research, vol. 6, pp. 1345-1382, 2005.

[29] S. Zhong, "Efficient online spherical k-means clustering," in IEEE International Joint Conference on Neural Networks, vol. 5, 2005, pp. 3180-3185.

[30] I. McCowan, J. Carletta, W. Kraaij, S. Ashby, S. Bourban, M. Flynn, M. Guillemot, T. Hain, J. Kadlec, V. Karaiskos et al., "The AMI meeting corpus," in Proceedings of the 5th International Conference on Methods and Techniques in Behavioral Research, vol. 88, 2005, p. 100.

[31] E. Gonina, G. Friedland, H. Cook, and K. Keutzer, "Fast speaker diarization using a high-level scripting language," in IEEE Workshop on Automatic Speech Recognition and Understanding (ASRU), 2011, pp. 553-558.

[32] M. S. Cracolice and J. C. Deming, "Peer-led team learning," The Science Teacher, vol. 68, no. 1, p. 20, 2001.

[33] J. H. L. Hansen, H. Dubey, and A. Sangwan, "CRSS-LDNN: Long-duration Naturalistic Noise Corpus Containing Multi-layer Noise Recordings for Robust Speech Processing," The Journal of the Acoustical Society of America, vol. 176th Meeting of Acoustical Society of America, Victoria, BC, Canada, p. to appear, 2018.

[34] "NIST DER script for RT evaluations," (Date last accessed 9Jan-2018). [Online]. Available: as part of the Speech Recognition Scoring Toolkit (SCTK): ftp://jaguar.ncsl.nist.gov/pub/sctk-2.4. 10-20151007-1312Z.tar.bz2

[35] "The 2009 (RT-09) Rich Transcription Meeting Recognition Evaluation Plan." [Online]. Available: https://web.archive.org/web/20100606092041if_/http: //www.itl.nist.gov/iad/mig/tests/rt/2009/docs/rt09-meeting-evalplan-v2.pdf

[36] "First DIHARD Challenge-Scoring tool, 2018." [Online]. Available: https://github.com/nryant/dscore 